\title{
Reporte de casos: bloqueo de erectores espinosos (ESP) en cirugía torácica pediátrica
}

Sandra Lataste', Alvaro León', Carolina Campillay

Introducción: Se presentan dos casos clínicos de menores de 5 años sometidos a cirugía torácica, en los que se utilizó como técnica analgésica post operatoria el bloqueo ESP. Se obtuvo muy buenos resultados respecto a dolor post operatorio, recuperación clínica del paciente, expansión torácica post quirúrgica y tolerancia a kinesioterapia precoz. 